\title{
Caregiver`s Gaze and Field of View Presumption Method During Bath Care in the Elderly Facility
}

\author{
Akiyoshi Yamamoto ${ }^{1}$, Noriyuki Kida ${ }^{2}$, Akihiko Goto ${ }^{3}$, Tomoko Ota ${ }^{4}$, \\ Tatsunori Azuma ${ }^{1}$, Syuji Yamamoto ${ }^{1}$, Henry Barrameda $\mathrm{Jr}^{1}$ \\ ${ }^{1}$ Super Court Co., Ltd, Osaka, Japan \\ yamamoto@city-estate.co.jp, \\ \{azuma, osakajyokoen, henrybarramedajr\} @supercourt.co.jp \\ ${ }^{2}$ Kyoto Institute of Technology, Kyoto, Japan \\ kida@kit.ac.jp \\ ${ }^{3}$ Osaka Sangyo University, Osaka, Japan \\ gotoh@ise.osaka-sandai.ac.jp \\ ${ }^{4}$ Chuo Business Group, Osaka, Japan \\ tomoko.ota@k.vodafone.ne.jp
}

\begin{abstract}
Reduced mental fatigue and ease of mind on caregivers are crucial in order to deliver safe bath care assistance in the elderly facility. In this paper, we present an experiment quantifying the eye gaze and field of view of the caregiver while performing bath care assistance. First, we used optical motion analyzing apparatus, head mounted gaze measuring apparatus, motion sensor (applied to 5 points: wrist, waist, neck, head and ankle in sequence) and compared data. Subjects imitated the motions of bathing assistance in a laboratory. Second, we clarified the validity of data by studying simultaneously recorded multiple people's data taken under role play settings (caregiver role and care receiver role) in an actual bathroom setting. The findings of the study are highly relevant in outlining a safe and secure bathing assistance related to reducing the mental burden on caregivers as well as giving them "ease of mind" while performing bath care.
\end{abstract}

Keywords: caregiver, elderly facility, bath care assistance, presumption method, blind spot, motion capture.

\section{Research Background and Problem Identification}

\subsection{Nursing Care for the Elderly}

Japan faces a critical need for nursing care as its elderly population continues to grow along with the rise of the number of elderly people who are bedridden, suffering from dementia, and of those requiring extended care. According to the 2013 White Paper on the Aging Society, 29.67 million people, or $23.2 \%$ of the entire Japanese population of 127.76 million, are senior citizens over the age of 65 . That number is well above the $7 \%$ threshold that defines a country as an aging society. Furthermore, a very high $11.8 \%$ of the population is over the age of 75 . 
The aging of society has increased demand for nursing care, with 5.49 million people as of October 2012 qualifying for some type of care in their daily life. This pressing social need necessitated specialized care in the nursing business. In 1987, the Certified Social Workers and Certified Care Workers Act was enacted to provide and maintain quality nursing care, giving specialized nursing care providers with national certification.

This also had the effect of directing the spotlight on the overwhelming challenges of the families dealing with nursing care for their elderly, and a growing call for socialized nursing care led to the enactment of the long-term care insurance program in 2000, giving recipients a choice in the type of nursing care they receive. The first article of the long-term care act advocates for socially supported quality welfare services that recognize the right to a dignified life for all individuals. It states that" The purposes of this Act are to improve health and medical care and to enhance the welfare of citizens. With regard to people who are under condition of need for longterm care due to disease, etc., as a result of physical or emotional changes caused by aging, and who require care such as for bathing, bodily waste elimination, meals, etc., and require the functional training, nursing, management of medical treatment, and other medical care, these purposes are to be accomplished by establishing a long-term care insurance system based on the principle of the cooperation of citizens, solidarity, and determining necessary matters concerning related insurance benefits, etc., in order to provide benefits pertaining to necessary health and medical services and public aid services so that these people are able to maintain dignity and an independent daily life routine according to each person's own level of abilities."

\subsection{Bathing Assistance: Its Background and Issues}

Bathing is indispensable for both hygiene and as part of Activities of Daily Living (ADL). It encourages blood circulation and promotes higher metabolism. It also relaxes the muscles, helps prevent bedsores and infections, regulates bowel movements and supports other such functions of the body. For many elderly people suffering from anxiety and tension, bathing is an important time for them to relax. It has the added mental benefit for the elderly, who consider bathing one of life's pleasures. Furthermore, cleansing the body of dirt and odors helps strengthen interpersonal relationships and encourages active social engagement. Bathing is thereby an extremely significant way to assist in the daily lives of the elderly, and in order to realize quality welfare service, effective bathing assistance is a necessity.

Bathing has many benefits -- physiological, mental and social. And yet, it can be risky, fraught with such dangers as falling and drowning. The external causes of bathing accidents include exposure to the cold while dressing/undressing, the warm temperature in the bathtub or bathroom, and the hydrostatic pressure within the bathtub are considered to have a negative impact on the bodies of the elderly. In many cases, the elderly tend to suffer from multiple complications, such as high blood pressure and diabetes, as well as arteriosclerotic changes and a deteriorating autonomic nervous system response. Therefore, some of the internal causes of bathing accidents can be attributed to changes in blood pressure, dehydration and blood coagulation. 
These physiological changes expose the elderly to a higher risk of cardiac arrest, cerebrovascular disorder, dizziness and cataleptic attacks. A complex mix of these factors heightens the risk of falling and injury, drowning and even death. Delivering quality bathing assistance depends on overcoming variety of issues.

Assisting with bathing is extremely hard work and has been an issue at welfare facilities for the elderly. There have been numerous reports published about the level of burden incurred while performing tasks during bath care assistance (Fujimura, 1995). One of them (Nagata, 1999) involves a survey of caregivers who work in the nation's 969 special nursing home for the elderly. According to the survey, the most physically taxing aspect of care is bathing assistance, followed by diaper change and transfers. The burden incurred by bathing assistance is threefold: physiological, physical and mental. The high temperature in the bathroom is demanding on the caregiver's body, and it causes physiological challenges. According to a study by Kawahara and his team (2010), caregivers showed high cortisol levels -- a benchmark for stressresponse -- after assisting with bathing, linking the activity with high physiological stress.

The high stress level in handling a lot of transfers from one place to another or changing position like transferring from the wheelchair to the bathtub and back, as well as supporting the elderly in an upright position while he or she dresses or undresses in a high-temperature bathroom can be compared to working in an assemblyline style work environment.. These are all tough on the back. Bathing assistance in a nursing care facility with a large bath involves not only horizontal, but lots of vertical movements that impact and stress the musculoskeletal structure, which in turn invites fatigue. Also, with the progressive bathing system most widely used in care facilities, caregivers end up with five manual transfers of an elderly from one location to another, including the move from the bedroom to the bathroom, which again causes stress to the caregiver.

Because bathroom floors could be slippery and may trigger a fall, constant vigilance can cause mental stress to caregivers. According to a survey by Nagata (1999), $18 \%$ of the caregivers cited "fear of a fall" as their biggest mental stress while assisting with bathing. Furthermore, the survey revealed that bathing assistance is the most conducive to feeling negative about nursing care (Kawahara 2010).

As detailed above, bathing assistance is extremely taxing work and is believed to be one of the main reasons why some people leave the job. The turnover rate for care jobs was $18.7 \%$ in 2008 , which is high compared against the industry's rate of $14.6 \%$. With regards to active job-opening ratios, the number declined to 1.34 in September 2009, after peaking at 2.53 in December 2008. To this day, the ratio continues hovering above 1 -- a big issue to consider in delivering quality care.

\subsection{Research Trends in Bathing Assistance}

To tackle the above-mentioned problems, fundamental and practical research is being done, with some of the research showing promise of mitigating the physical toll of bathing on the elderly. For instance, Kanda (1991) published the results of his research involving the physiological burdens of bathing in the winter and in the 
summer. Nagahiro's research (2006) on the impact of bathroom temperatures on the circulatory system of healthy elderly people shows temperature levels that promote lower blood pressure after bathing.

On the other hand, numerous studies conducted in the areas of sanitary engineering and structural engineering have contributed to the development of comfortable clothing and structural improvements in buildings to reduce the physiological impact of care-giving on care workers.

In terms of dealing with the physical stress, caregivers are provided with ergonomic and biomechanic suggestions and nursing equipment that help reduce back strain. For instance, when comparing the efficiency between the use of a mechanical lift and a manual lift when transferring a fully dependent care receiver to a wheelchair, a Tomioka study (2008) showed that the mechanical lift reduced the work time considerably after a period of training and that the mechanical lift was effective in reducing the caregivers' back strain. Furthermore, after analyzing the burden on the back by studying the angle of the upper body and the results of electromyogram, the Tomioka report (2007) showed that using the mechanical lift to get in and out of the bathtub reduced the caregiver's forward-leaning posture and strain on the muscles -- all of which contributed to an overall reduction in work-related stress. The report also pointed to increased back strain that comes from bending forward when the caregiver has to wash a care receiver or help her dress/undress while she remains seated, or when adjusting the footrest on the wheel chair.

\subsection{Identifying the Problem}

There's been a lot of research done for the purpose of lessening the physiological and physical burdens for both the caregivers and the care receivers, many of them outlining specific nursing techniques and new policies. However, when it comes to easing the mental burdens, basic research doesn't go far enough, and there are as yet no solutions.

Bathing assistance is more prone to accidents, the caregiver must be on constant alert, especially when dealing with elderly with dementia, who often move and act in unpredictable ways. Creating a safe way to offer bathing assistance that can prevent falls or drowning accidents is in a way reducing mental stress. This could achieve "peace of mind," and make quality nursing care a reality.

We have identified the main issue in our research as reducing the caregivers' mental stress by preventing accidents, such as falls and drowning.

\section{Preventing Accidents during Bathing Assistance}

\subsection{Causes of Falls: Care Receivers' Physical Issues}

Kawamura (2003) pointed out that medical incidents surrounding nursing care mostly involve falls. Not just limited to bathing time, falling accidents by elderly people can severely interfere with their health, for instance forcing them to be bedridden. A large number of studies have been conducted on the link between fitness levels and falling 
accidents of an elderly person. Accident-prevention programs based on those findings have been shown to be effective.

Falls can be caused by many factors: old age, disease, loss of muscle strength and deterioration of other physical functions of the care receiver. Therefore, to prevent a fall, the report said that one must assess the risks care receivers are exposed to and design a preventative program.

\subsection{Causes of Falls: Environmental Factors}

The causes of falls are not limited to declining physical functions of the care receivers: steps, slippery floors, footwear and poor lighting are also culpable. These are referred to as environmental factors and can be addressed to prevent falls. Ergonomic research -- through the quantitative studying of the positioning, line of vision and visual field of train conductors, automobile drivers and other people who work in dangerous environments -- has done much to contribute to safety policies. The study has led to the development of a technology that can detect vehicles within a blind spot by anticipating when a vehicle in the opposite lane enters into a driver's blind spot. Blind spots occur where the driver cannot see an object that also does not show up in the rearview and side view mirrors. The larger the vehicle, the larger the blind spot. Accidents are more likely to happen when the driver fails to see objects in the blind spot. By warning the driver of a vehicle within the blind spot, the driver can reduce his chances of getting into an accident.

On the other hand, nursing care takes place where people, labor, machines and the environment come together harmoniously. It is an extremely difficult line of work where caregivers have to respond to sudden and unexpected actions of the care receivers with flexibility. Furthermore, chronic staff shortages at care sites mean caregivers do not have the luxury to foster harmony between the people and their environment, and the environment is not set up to take better care of the caregivers themselves; blind spots are everywhere. With regards to research on blind spots in care sites, most are focused on positioning video cameras in places that reduce these blind spots. In the area of care giving, it is hoped that the technological development is based on ergonomics, promoting independent living and normalization, general respect and respect toward basic human rights, self-actualization and other crucial principles of welfare. Therefore, research on blind spots -- such as one that involves the use of video cameras -- must take into consideration the rights of the caregivers before launching into quantifying blind spots that occur daily in the care line of work.

Research into quantifying blind spots has just begun. Quantifying blind spots during bathing assistance, or ideas on where and how caregivers and receivers can position themselves to minimize those blind spots -- these are all areas for potential growth.

\subsection{Predicting and Safeguarding against Falls}

Elderly people, who form the majority of care receivers, suffer from a deterioration of various physical and mental functions. Therefore they require assistance to bath safely and with peace of mind. That means caregivers have to be able to predict a care 
receiver's set of risks and improve on their ability and judgment to draw information from multiple sources and act accordingly. In other words, when it comes to preventing bathing accidents from happening, it is imperative that caregivers are able to sense the receiver's mood, and assess the local environment. This also means that caregivers need the skill to sense danger and be able to act on it accordingly.

It is said that our sense of danger is grasped mainly through our vision -- more than $80 \%$ out of all five of our senses. When caregivers and receivers come together during a bath and the caregiver uses her vision to sense danger, the caregiver should either consciously or subconsciously know where he or she is looking to perceive that danger and turn that into data of sorts. But there has yet to be an objective evaluation of visually extracted information at care sites. Plus, it isn't clear where a caregiver should look and what to look for in order to effectively sense danger. To predict a fall during a bath requires a superior level of observation, judgment and then on top of that, the ability to handle it with flexibility. These types of skills are not acquired in a short amount of time; it requires years of experience and learning.

Also, caregivers try not to interfere with the activities of the care receivers as much as they can to encourage their independence. At care sites, the idea of "monitoring" is of paramount significance. Even when monitoring during bathing assistance, where the caregiver should look (for danger signs) has not yet been quantified. It has been a nursing technique acquired mostly through intuition and experience.

\subsection{Solving Problems}

This research recommends that in order to prevent a fall during a bath, a practical system be instituted whereby neither a caregiver's gaze nor the care receiver end up in a blind spot. For that to happen, the caregiver's and care receiver's location in the bathroom or changing room has to be recorded in a timed series. As for the caregivers, tracking their gaze in a timed series can help quantify blind spots, potentially even turning the caregiver's monitoring skill into data.

\section{Predicting Positions and Field of Vision}

\subsection{Motion Capture}

With respect to measuring physical exercise and positional information, the most commonly used system is the DLT motion-capture system, which primarily uses visual data. In a motion-capture system, an optical motion measuring apparatus is used, and multiple infrared cameras are used to measure the three-dimensional coordinate of a marker. This system is capable of accurately measuring a three-dimensional coordinate and is extremely expensive, but its use is limited to water-free locations.

For compiling eye-gaze data, an eye-gaze tracking device that uses pupil corneal reflection technique is mounted on the head. This technique can accurately track the eye gaze, but the device is extremely expensive and raises some privacy issues as it records the image of the care receiver taking a bath. 
The use of motion-capture systems and head-mounted eye-gaze tracking devices can be problematic and difficult to justify. They are large and intrusive, and raise privacy issues.

\subsection{Estimating by Using Motion Sensors}

In recent years, accelerometers and GPS sensors have become standard features in mobile phones. In much the same way, motion sensors such as accelerometers, gyro sensors and geomagnetic sensors have become compact and affordable due to the MEMS technology. Motion sensors, which are attached to different parts of the body, can read bodily rotations and translational motion in a simple and unrestricted way. Because it doesn't require video images to capture positioning, eye-gaze and visual field data, the technique is highly anticipated, even from a privacy standpoint.

Since data retrieved from motion sensors are accelerated, posture data, which is an important element in measuring physical exercise, can be attained by the conversion of the angular velocity's integration operator or the gravitational acceleration.

However, when taking the angular velocity through integral value calculation, drift errors contained in the power output of gyro sensors compound and lower the accuracy of the outcome the longer the measuring process. Furthermore, converting from gravitational acceleration doesn't provide information on direction, and with passing time, the acceleration sensor reads the dynamic acceleration as error. The gyro sensor's drift error can be corrected in a number of suggested methods -- gyro sensors, accelerometers, algorithms that predict roll, pitch and yaw angles from geomagnetism sensors, as well as algorithms that predict quaternions.

Therefore, we suggest instituting a system by which the caregiver's gaze can be quantified at an actual bathing site. The goal is to reduce the mental burdens at care sites by delivering a safe way to assist with bathing and creating "peace of mind." This system has the ability to quantify assisting skills and blind spots, and monitor activity while assisting with bathing. It can also evaluate staff assignments objectively. In order to make this system a reality, what is necessary as part of basic research is the verification of the accuracy of positioning, gaze and field of view data acquired through motion sensors.

\section{$4 \quad$ Suggested Systems}

\subsection{System Outline}

This is a system that uses motion sensors to estimate position and visual field data. Calculating the caregiver and the care receiver's position within $10 \mathrm{~cm}$ of accuracy in the bathroom, then making an estimate of the caregiver's field of view data in the direction that the caregiver is facing (front) is thought to be helpful for the caregiver. Position estimation by motion sensor is not new. However, methods for estimating visual fields have yet to be tested and are considered something of a novelty. By dispensing with the use of videos in the process, the system makes no privacy breaches, making it a strong candidate for use in welfare care studies, psychology, ergonomic 
and other areas of research. Furthermore, the skills caregivers apply during bathing -assisting and monitoring -- have always been ambiguous. Quantifying those skills from gaze data offers an unprecedented viewpoint. This research is being used for system development intended as feedback to care sites and has a practical application: reducing mental burden is effective in improving the work environment of care sites.

Using this system will more clearly reveal the difference in bathing assistance and monitoring skills between veteran caregivers and novice caregivers. The system can eventually develop into one that supports skill development and offers assessments that can help with monitoring skills to better assist with bathing. The existence of blind spots in bathing assistance work has been made clear, paving the way for smarter staff appointments. Clarifying policies that promote safe bathing assistance by observing the location and movements of the caregivers and care receivers also contributes to the safe management of care welfare facilities.

\subsection{Basic Experiment}

The experiment aimed at clarifying the reliability and validity of motion-sensor data - as it is conducted as an experiment in a laboratory -- based on the three-dimensional coordinate data derived from optical motion measurement equipment. It was conducted on general subjects at a laboratory. In the experiment, four sets of infrared cameras (Raptor-H, Hawk-I, made by Motion Analysis Corp.) and a real-time optical motion capture system (MAC 3D System made by Motion Analysis Corp.) were used to collect three-dimensional data from four reflective marker points mounted on to the subjects' heads (Fig.1). As for the motion sensors, small sensors (Fig.2, LP-WS1201, Logical Product) consisting of a 3-axis accelerometer, a 3-axis angular velocity sensor, a 3-axis geomagnetism sensor and a GPS sensor were fixed to the subjects' heads and the data was collected at $1000 \mathrm{~Hz}$. The subjects were allowed to walk around and look around (Fig.3). By integrating the motion-sensor data, the subjects' body position and orientation were computed. And then their location, base and field of view information were estimated (Fig.4). As a result, movements and body orientations that are prone to estimation errors as well as places to attach motion sensors that produce the least amount of estimation errors have been made clear.

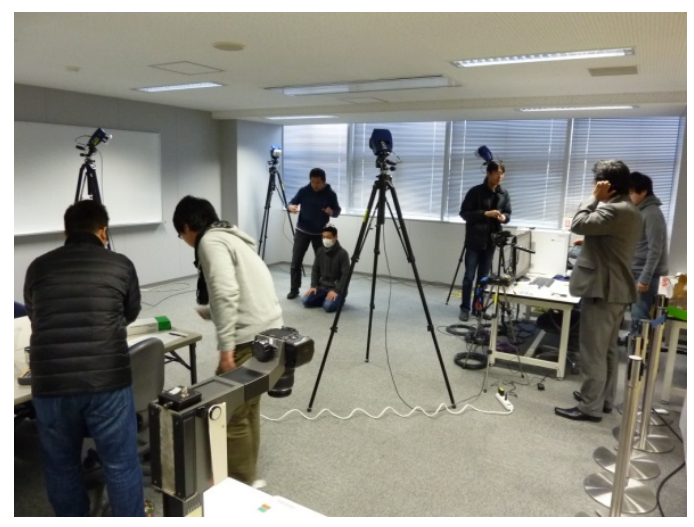

Fig. 1. Experiment in a laboratory setting 


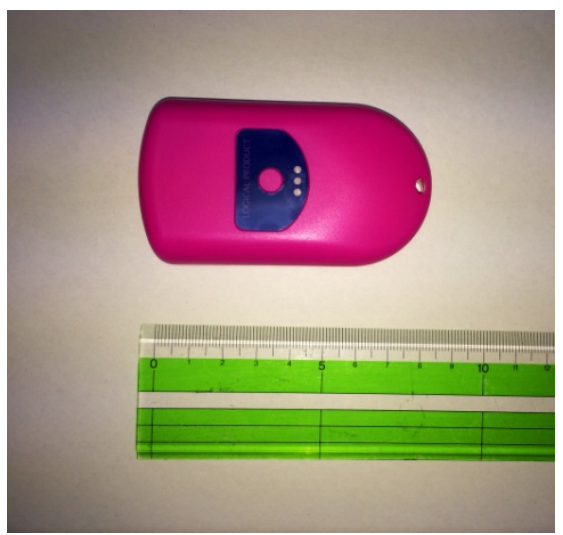

Fig. 2. Logical Product LP-WS1201 Wireless Motion Sensor

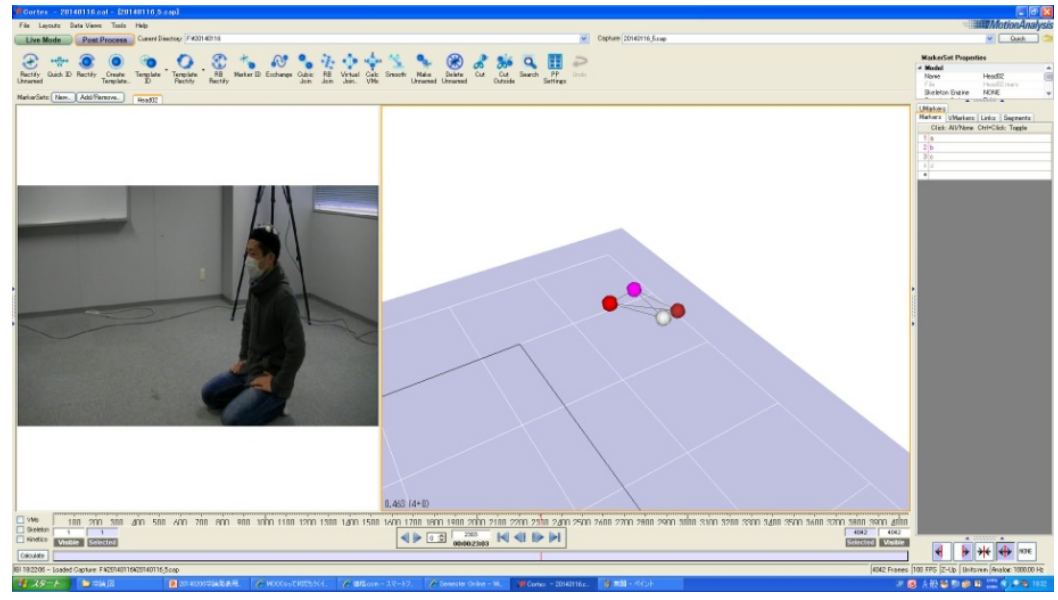

Fig. 3. Motion Capture Configuration Screen

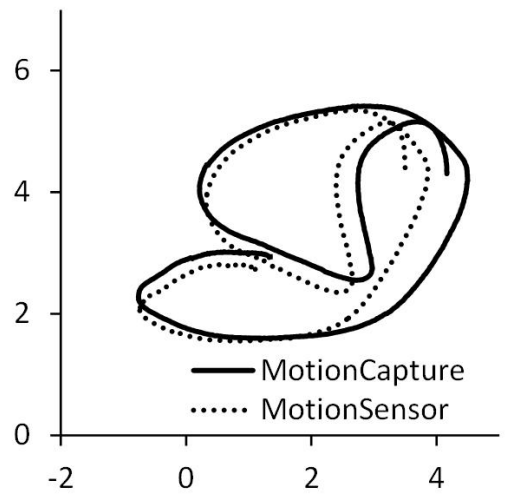

Fig. 4. Locomotion Trajectory in Horizontal Plane 


\section{Conclusion}

Reduced mental fatigue and ease of mind on caregivers are crucial in order to deliver safe bath care assistance in the elderly facility. In this paper, we presented an experiment quantifying the eye gaze and field of view of the caregiver while performing bath care assistance. We used optical motion analyzing apparatus, head mounted gaze measuring apparatus, motion sensor and compared data. Subjects imitated the motions of bathing assistance in a laboratory. As a result, movements and body orientations that are prone to estimation errors as well as places to attach motion sensors that produce the least amount of estimation errors have been made clear. The findings of the study would be highly relevant in outlining a safe and secure bathing assistance related to reducing the mental burden on caregivers as well as giving them "ease of mind" while performing bath care.

\section{References}

1. Fujimura, T.: Roujin Ho-mu ni okeru Kaigo Sagyou no Mondaiten to Youtsuu Taisaku. Roudou no Kagaku 509, 13-16 (1995) (in Japanese)

2. Nagata, H.: Tokubetsu Yougo Roujin Ho-mu de no Kaigo Roudou no Jittai Chousa to kongo no Kourei Kaigo Roudou no Kentou. Roudou Kagaku 75, 459-469 (1999) (in Japanese)

3. Kawahara, Y.: Current State of Bathing Care and Necessity of New equipment for Bathing Care Institution. J. Human and Living Environment. 171, 23-30 (2010)

4. Kanda, K.: Koureisha no Touki to Kaki ni okeru Nyuuyoku Kankyou to Nyuuyokuji no Seiriteki Futan ni Kansuru Chousa. Bull. Inst. Public Health 40, 388-390 (1991)

5. Nagahiro, C.: Effects of Room Temperature on Circulatory Dynamics During Bathing in the Elderly. Japanese Journal of Public Health 533, 178-186 (2006)

6. Tomioka, K.: Low Back Load and Satisfaction Rating of Caregivers and Care Receivers in Bathing Assistance Given in a Nursing Home for the Elderly Practicing Individual Care. San Ei Shi 49, 54-58 (2007) (in Japanese)

7. Tomioka, K.: Low Back Pain among Care Workers Working at Newly-built Nursing Homes for the Aged. San Ei Shi 50, 86-91 (2008) (in Japanese)

8. Kawamura, H.: Hiyari Hatto: 11,000 Jirei ni yoru Era- mappu Kanzenbon. Igaku shoin, 88-91 (2003) (in Japanese) 